\title{
Novel Cubic Magnetite Nanoparticle Synthesis Using Room Temperature Ionic Liquid
}

\author{
M. SUNDRARAJAN* AND M. RAMALAKSHMI \\ Department of Industrial Chemistry, \\ School of Chemistry, Alagappa University, \\ Karaikudi - 630003 , \\ Tamil Nadu, INDIA. \\ sundrarajan@yahoo.com
}

Recieved 11 September 2011; Accepted 8 November 2011

\begin{abstract}
Room temperature ionic liquids are relatively more useful in the synthesis of inorganic nanostructured materials because of their unique properties. To synthesize the iron oxide nanoparticle in simple precipitation method, a novel ionic liquid was used as the greener medium and stabilizing agent namely "1-n-butyl-3-methylimidazolium trifluoromethane sulfonate [BMIM][TfO]". The crystallinity, chemical structure, morphology and magnetic properties of the synthesized magnetite nanoparticles have been characterized by using X-ray diffraction (XRD), Fourier Transform Infrared spectroscopy (FT-IR), Scanning electron microscopy (SEM), Atomic force microscopy (AFM), Transmission electron microscopy (TEM) and Vibrating sample magnetometer (VSM) studies. The XRD study is divulge that the synthesized magnetite nanoparticles have inverse spinel face centered cubic structure. The FT-IR vibration peaks show the formation of $\mathrm{Fe}_{3} \mathrm{O}_{4}$ nanoparticles, where the vibration peak for $\mathrm{Fe}-\mathrm{O}$ is deliberately present at $584 \mathrm{~cm}^{-1}$. The average particle size of the synthesized nanoparticles is found to be $35 \mathrm{~nm}$. Homogeneously dispersed cubic shape with superstructure is found through SEM, AFM and TEM examination studies. The synthesized iron oxide nanoparticles have a high saturation magnetization value of $25 \mathrm{emu} / \mathrm{g}$, which is very much useful for biomedical applications.
\end{abstract}

Keywords: Magnetic properties, Nanoparticles, Ionic liquid, X-ray diffraction, Magnetic materials.

\section{Introduction}

Room temperature ionic liquids (RTILs) are salts with low melting points that exist as liquid typically below $100^{\circ} \mathrm{C}$. Ionic liquids are used as solvent for the synthesis of nanoparticles, and attracted lot of attention in the recent years ${ }^{1}$. In comparison with conventional organic solvents, ionic liquids can have multi-universal properties such as high fluidity, low melting point, extended temperature range in liquid form, air and water stability, low toxicity, nonflammability, high ionic conductivity, very low vapor pressure and ability to dissolve a 
variety of materials ${ }^{2}$. Ionic liquids act as a "nanosynthetic template" to stabilize the metal and metal oxide nanoparticles and also provide an electrostatic protection in the form of a "protective shell" for nanoparticles ${ }^{3}$. Now-a-days, imidazolium based ionic liquids have been used as eco-friendly alternative reaction media for the conventional volatile organic solvents for the reaction acceleration purposes ${ }^{4}$.

Magnetite nanoparticle have cubic inverse spinel structure with oxygen forming a FCC closed packing and Fe cations occupying both the interstitial tetrahedral and octahedral sites $^{5}$. In the recent years, magnetic nanoparticles have been effectively studied because of their fascinating properties for biological applications especially for magnetic drug targeting, tags for biomolecular sensors, biomolecule separation and magnetic resonance imaging (MRI) contrast agents ${ }^{6-11}$. During and after synthesis of magnetic nanoparticles, are tend to agglomerate and form bigger particles. The coating is used to stabilize the particles in colloidal form, to prevent them from degradation and to minimize the toxicity. Generally, magnetite and maghemite are the two important forms of iron oxide, which are used as the magnetic materials for biomedical applications. Agglomeration of magnetic nanoparticles is inevitable, because of their Vander Waals attractive forces between the tiny particles ${ }^{12}$. Magnetite $\left(\mathrm{Fe}_{3} \mathrm{O}_{4}\right)$ nanoparticles are chemically stable and non-carcinogenic and has high saturation value $(92 \mathrm{emu} / \mathrm{g})$ compared to the maghemite bulk material $(82 \mathrm{emu} / \mathrm{g})^{13-15}$.

In this work, we explored the facile route synthesis of magnetite nanoparticles using 1n-butyl-3-methylimidazolium trifluoromethane sulfonate [BMIM][TfO] ionic liquid. Unlike the nanoparticles synthesized by other methods, the synthesis of the iron oxide nanoparticles in our work was carried out only by the precipitation of ferrous salt in alkaline aqueous solution at low temperature. Here, the [BMIM][TfO] ionic liquid was used as the solvent and stabilizer to efficiently tune the particle formation, to prevent the interparticle aggregation and to regulate the shape of the iron oxide nanoparticles. A novel room temperature ionic liquid - assisted method is more facile and effective to synthesize $\mathrm{Fe}_{3} \mathrm{O}_{4}$ magnetite nanoparticles at desired level.

\section{Material and Methods}

All chemicals used in this work were of analytical grade, purchased from E-Merck and used as received without further purification. A $0.75 \%$ [BMIM][TfO] and $0.1 \mathrm{M}$ ferrous sulfate heptahydrate solutions were prepared by using double distilled water. They were mixed and vigorous stirring was made for $30 \mathrm{~min}$. After stirring, the reaction mixture was placed in a preheated water bath $\left(70^{\circ} \mathrm{C}\right)$. A concentrated solution of sodium hydroxide was quickly added into the mixture to bring the $\mathrm{pH} 11$ and subsequently stirred for an hour. After mixing the $\mathrm{NaOH}$ solution, the color of the solution changed was from pale green to black, indicating the formation of $\mathrm{Fe}_{3} \mathrm{O}_{4}$ nanoparticles. The prepared nanoparticles were magnetically separated and the ionic liquid was collected by decantation. The obtained magnetite nanoparticles were washed with distilled water and dried in vacuum oven at $80^{\circ} \mathrm{C}$ for $8 \mathrm{~h}$.

X-Ray Diffraction (XRD) pattern was recorded on a PANlytical X'Pert PRO Diffractometer with $\mathrm{K} \alpha$ radiation $\left(\lambda=1.5406^{\circ} \mathrm{A}\right)$. FT-IR spectrum was taken for the synthesized nanoparticles using a SHIMADZU-8400-S spectrometer in the range of 4000$400 \mathrm{~cm}^{-1}$ using $\mathrm{KBr}$ pellet. The surface morphology of the synthesized nanoparticle was observed through SEM (HITACHI S-3000H). For AFM analysis, the nanoparticle suspension was diluted with distilled water 1:2500 and dropped onto a surface of unruffled mica. After air drying, probes were scanned with a DualScope C26 (DME, Herlev, Denmark). Transmission electron microscopy (TEM) images were recorded on a JEOL JEM $2000 \mathrm{EX}$ microscope at an accelerating voltage of $200 \mathrm{Kv}$. The magnetic property of the 
prepared iron oxide nanoparticle was revealed with vibrating sample magnetometer at room temperature using VSM, Lakeshore7404.

\section{Results and Discussion}

The XRD pattern of the synthesized nanoparticle by using ionic liquid-assisted method is shown in Fig.1. The position and intensity counts of the diffraction peaks of the sample match well with the standard XRD data for bulk magnetite (JCPDS No. 88-0866). The characteristic peaks appeared at $30.2^{\circ}(220), 35.5^{\circ}(311), 43.1^{\circ}(400), 57.0^{\circ}(511)$ and $62.6^{\circ}$ (440) are can be assigned to the peaks of the $\mathrm{Fe}_{3} \mathrm{O}_{4}{ }^{16,17}$. The calculated lattice constant value of the sample was $8.39^{\circ} \mathrm{A}$, which is coincide with the lattice constant of magnetite $\left(8.384^{\circ} \mathrm{A}\right)$ but for the maghemite is $8.35^{\circ} \mathrm{A}$. Using Scherrer's formula, the crystallite size of the synthesized $\mathrm{Fe}_{3} \mathrm{O}_{4}$ nanoparticles was calculated and it is about $33 \mathrm{~nm}$. No peaks of any other phases are seen in this pattern, indicating that the high purity of the synthesized magnetite nanoparticles.

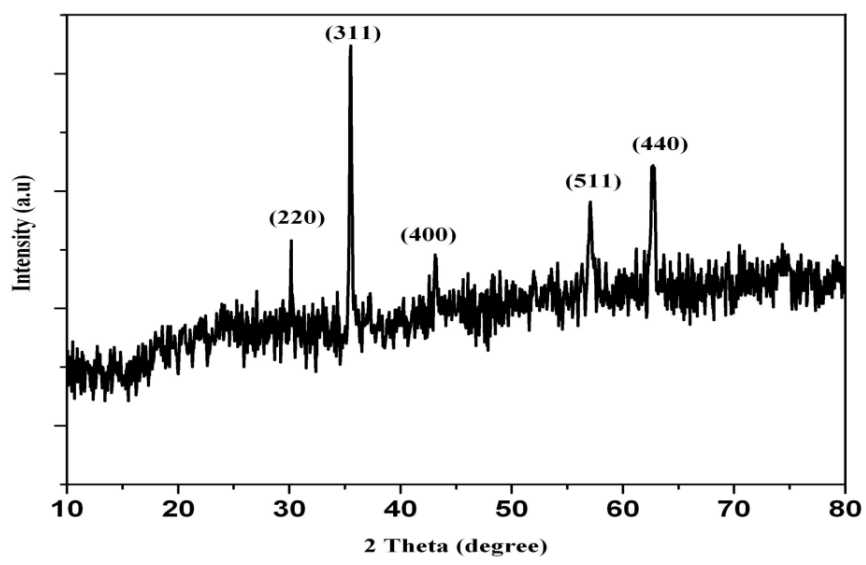

Figure 1. XRD pattern of synthesized magnetite nanoparticles using [BMIM][TfO] ionic liquid.

According to Z.H. Shou et al. ${ }^{18}$, the formation of $\mathrm{Fe}_{3} \mathrm{O}_{4}$ is as follows:

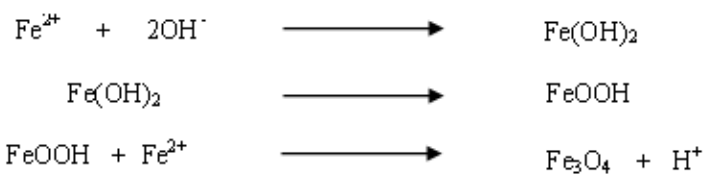

The FT-IR spectrum of the synthesized $\mathrm{Fe}_{3} \mathrm{O}_{4}$ magnetite nanoparticles using [BMIM][TfO] ionic liquid is presented in Fig. 2. The strong absorption band at $584 \mathrm{~cm}^{-1}$ is assigned to the vibrations of the $\mathrm{Fe}-\mathrm{O}$ bond, which confirm the formation of $\mathrm{Fe}_{3} \mathrm{O}_{4}$ nanoparticles. Previously, it was reported that the characteristic absorption band for $\mathrm{Fe}-\mathrm{O}$ in bulk $\mathrm{Fe}_{3} \mathrm{O}_{4}$ is appeared at 570 and $375 \mathrm{~cm}^{-1}$ wavenumber ${ }^{19}$. However, in the present case the band for Fe-O shift towards higher wavenumber of $584 \mathrm{~cm}^{-1}$. It is due to the breaking of the large number of bands for surface atoms, resulting rearrangement of localized electrons on the particle surface and the surface bond force constant increases as $\mathrm{Fe}_{3} \mathrm{O}_{4}$ is reduced to nanoscale dimension, so that the absorption bands shift to higher wavenumber ${ }^{20}$. The broad 
bands at $3410 \mathrm{~cm}^{-1}$ and $1640 \mathrm{~cm}^{-1}$ are assigned to $\mathrm{O}-\mathrm{H}$ stretching and bending vibrations of water respectively, which is present on the surface of the iron oxide nanoparticles ${ }^{21}$. Since there are large surface to volume atomic ratio, high surface activity and more dangling bonds on the nanoparticle surface, the atoms on the nanoparticle surface are apt to adsorb ions or molecules in solution. The $\mathrm{Fe}_{3} \mathrm{O}_{4}$ nanoparticles, in neutral aqueous solution, the bare atoms of $\mathrm{Fe}$ and $\mathrm{O}$ on the particle surface would adsorb $\mathrm{OH}^{-}$and $\mathrm{H}^{+}$respectively, hence there is $\mathrm{OH}$ rich surface. The $-\mathrm{OH}$ on the surface can easily react with any positive moiety to modify the surface. The band between 2640 and $3000 \mathrm{~cm}^{-1}$ are due to the imidazolium ring of the ionic liquid.

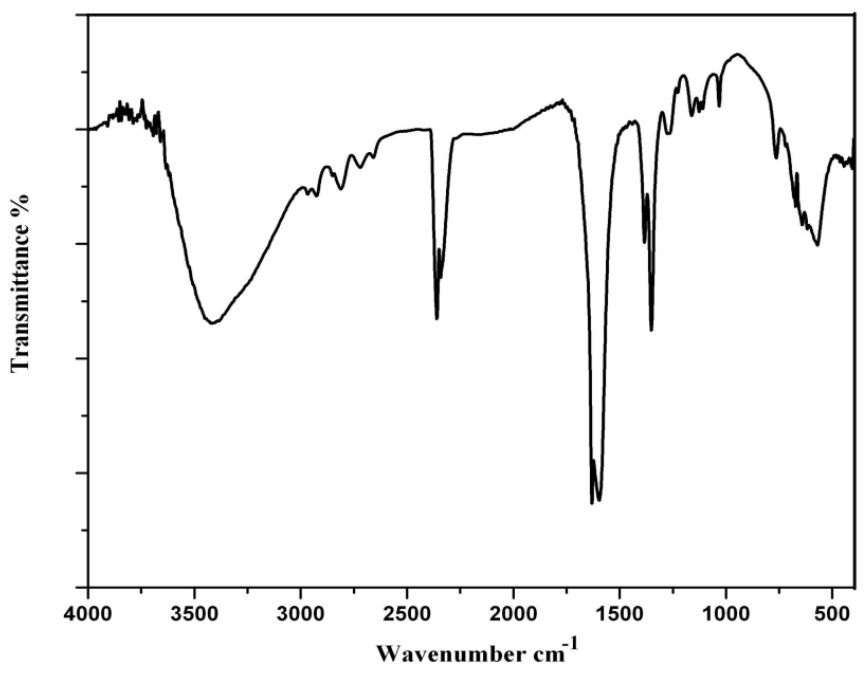

Figure 2. FTIR spectrum of synthesized magnetite nanoparticles using [BMIM][TfO] ionic liquid.

The size and shape of the $\mathrm{Fe}_{3} \mathrm{O}_{4}$ nanoparticles are characterized through SEM study, the micrograph is presented in Fig. 3. It can be seen that the particles have a large quantity of dispersive nanoparticles with the average size about $35 \mathrm{~nm}$. These nanoparticles are moderately uniform and appear as cubic structure.

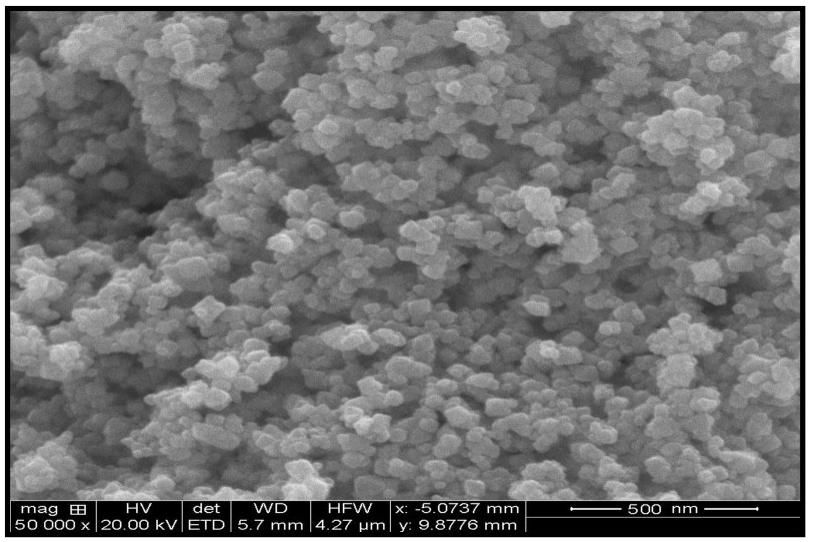

Figure 3. SEM micrograph of prepared magnetite nanoparticles. 
To investigate the morphology and dispersity of the synthesized nanoparticles, the AFM and TEM studies have been performed and the resulting micrographs are presented in Fig. 4 and 5. It can be seen that the particles have irregular distribution and the particle size is about $35 \mathrm{~nm}$. The TEM image is clearly depict the formation of superstructure on the magnetite nanoparticles. As evidenced by the XRD, AFM and SEM studies, the particles are in cubic shape and average particle size is about $35 \mathrm{~nm}$.
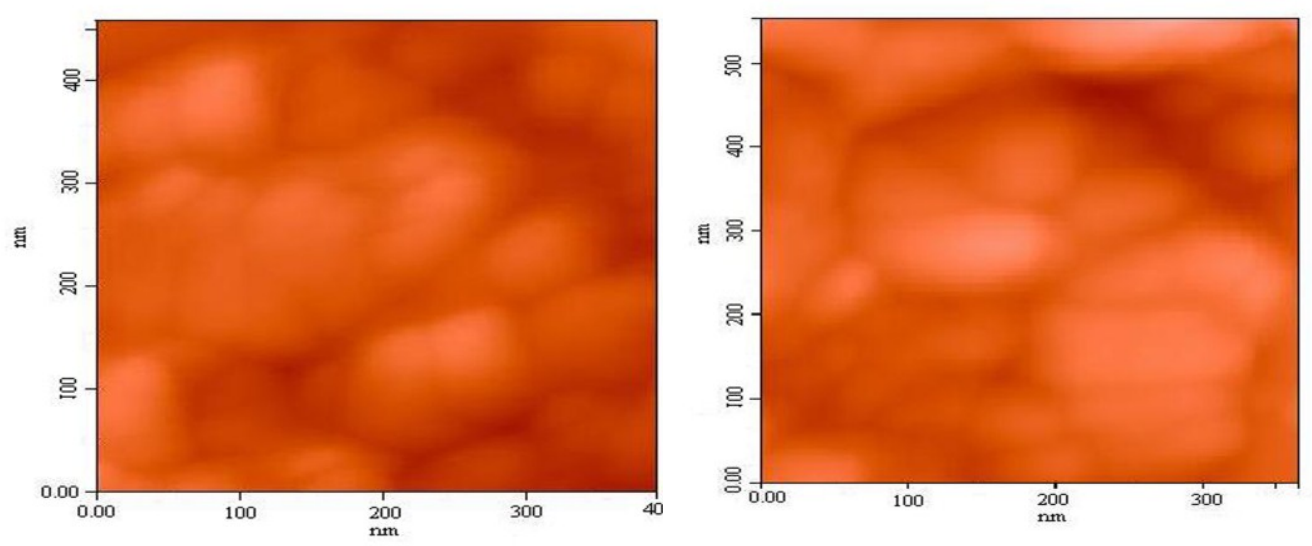

Figure 4. AFM photographs of synthesized Magnetite nanoparticles in [BMIM][TfO].

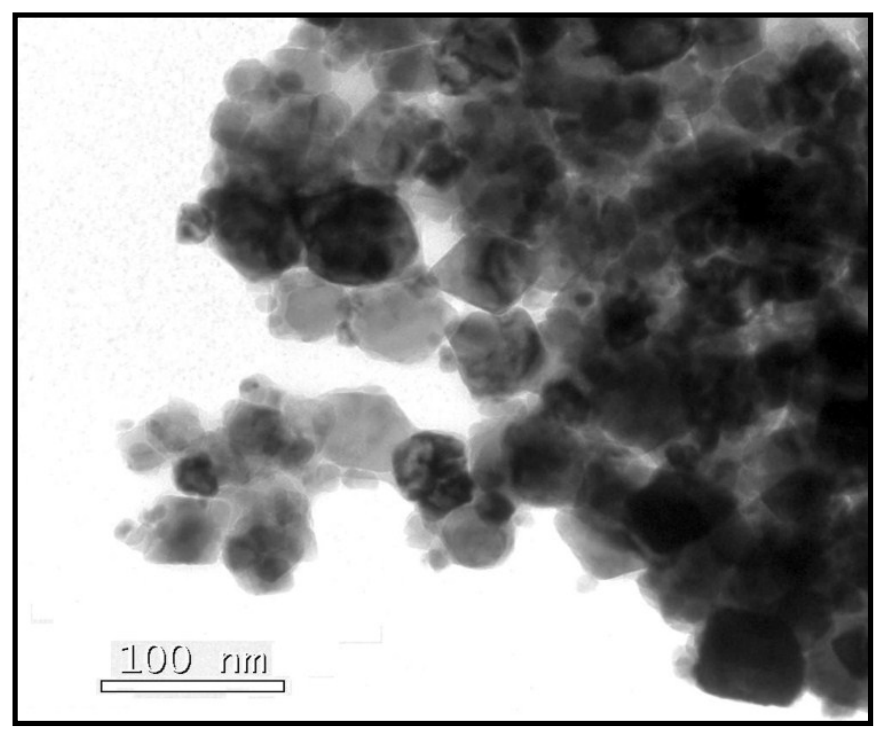

Figure 5. TEM micrograph of synthesized magnetite nanoparticles.

Fig. 6 is the hysteresis loop taken at room temperature for the synthesized magnetite nanoparticles. It can be seen that the nanoparticles are superparamagnetic with a coercivity 
of 296 Oersteds and the saturation magnetization is about $25 \mathrm{emu} / \mathrm{g}$, which is lower than the value of bulk magnetite $(92 \mathrm{emu} / \mathrm{g})$. The reduction in magnetization value is due to the decrease of magnetic nanoparticle size. This result is corroborate with results reported elsewhere $^{22}$.

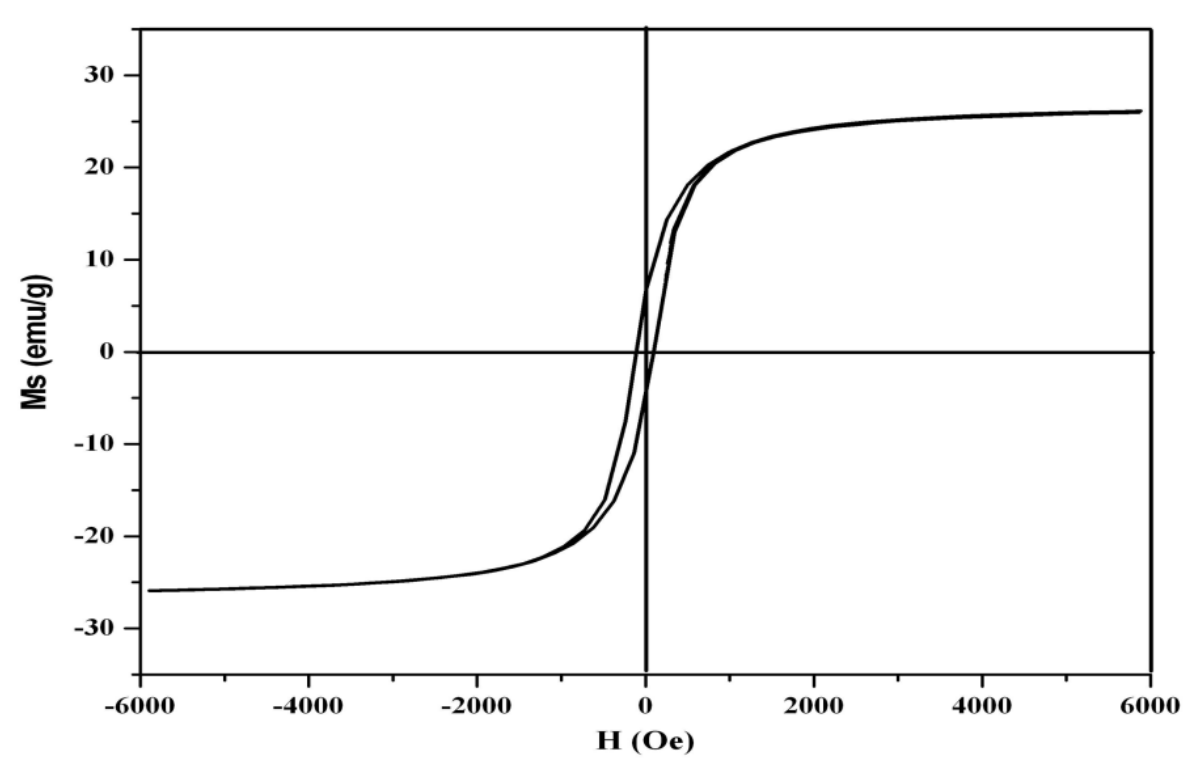

Figure 6. Hysteresis loop of synthesized magnetite nanoparticles using $[\mathrm{BMIM}][\mathrm{TfO}]$ ionic liquid at room temperature.

In our experiment, we could able to synthesize the cubic shape iron oxide magnetite nanoparticles whose size is about $35 \mathrm{~nm}$. The shape and size controlled product is able to synthesize using the [BMIM][TfO] ionic liquid owing to its large surface activity, and the reactants could disperse in ionic liquid which forms a lot of petite reactors. Room temperature ionic liquids have high polarity and interfacial tension which made the inorganic species to higher nucleation rate, which thrusted the formation of the nanoparticles ${ }^{2}$.

\section{Conclusion}

Present article, report a facile process for the preparation of cubic magnetite nanoparticles using [BMIM] [TfO] ionic liquid as a medium and stabilizer which is confirmed by the XRD study. The formation of $\mathrm{Fe}_{3} \mathrm{O}_{4}$ nanoparticles is confirmed by the FT-IR spectrum, where the presence of vibrational peak at $584 \mathrm{~cm}^{-1}$ is assigned for $\mathrm{Fe}-\mathrm{O}$ bond. The average diameter of the synthesized iron oxide nanoparticles is around $35 \mathrm{~nm}$. A higher magnetic saturation value of $25 \mathrm{emu} / \mathrm{g}$ is obtained for the synthesized $\mathrm{Fe}_{3} \mathrm{O}_{4}$ cubic magnetic nanoparticles which is obviously lower than the bulk value of $\mathrm{Fe}_{3} \mathrm{O}_{4}$ magnetite nanoparticles. The used ionic liquid can be recovered and reused for the subsequent reactions, so as the medium/solvent wastage can be minimized. The strategy adopted in this work can be followed for the preparation of nanostructures of various transition metal oxides. 


\section{References}

1. Wang Y and Yang H Chem. Eng. J. 2009, 147, 71.

2. Zou D, Xu C, Luo H, Wang L and Ying T Mater. Lett. 2008, 62, 1976.

3. Kramer J, Redel E, Thomann R and Janiak C Organometallics 2008, 27, 1976.

4. Kim D W, Song C E and Chi D Y J. Am. Chem. Soc. 2002, 102789.

5. Cornell R M and Schwertmann U VCH, New York, 1996, 28.

6. Ito A, Shinkai M, Honda H and Kobayashi T J. Biosci. Bioeng. 2005, 100, 1.

7. Papisov M I, Savelyev V Y, Sergienko V B and Torchilin V P Int. J. Pharam. 1987, 40, 201.

8. Schroder L, Lowrej T J, Hilty C, Wemmer D E and Pines A Science 2006, 314, 446.

9. Graham D L, Ferreira H A and Freitas P P Biotechnology 2004, 22, 455.

10. Safarik I and Safarikova M J J. Chromatogr. B. Biomed. Sci. Appl. 1999, 722, 33.

11. Mornet S, Vasseur S, Grasset F and Duguet E J. Mater.Chem. 2004, 14, 2161.

12. Tural B, Ozkan N and Volkan M J. Phys. Chem. Solids 2009, 70, 860.

13. Dallas P, Georgakilas V, Niarchos D, Komniou P, Dehagias T Nanotechnology 2006, 17, 2046.

14. Cannas C, Concas G, Gatteschi D, Musinu A, Piccalaga G and Sangregoria C J. Mater. Chem. 2002, 12, 3141.

15. Sivii C, Popviv M, Enache C, Subrt J, Niznansky D, Bakardzieva S, Caizer C and Hrieance I Solid State Ionics 2002, 151, 219.

16. Zhai Y, Liu F, Zhang Q and Gao G Colloids and Surfaces A: Physicochem. Eng. Aspects 2009, 332, 98.

17. Tural B, Ozkan N and Volkan M J. Phys. Chem. Solids 2009, 70, 860.

18. Zhou Z H, Wang J, Liu X and Chan H S O J. Mater. Chem. 2001, 11, 1704.

19. Waldon R.D Phys. Rev. 1955, 99, 1727.

20. Ma M, Zhang Y, Yu W, Shen $\mathrm{H}$, Zhang $\mathrm{H}$ and $\mathrm{Gu} N$ Colloids and Surfaces A : Physicochem. Eng. Aspects 2003, 212, 219.

21. Cai W and Wan J J. Colloid Interface Sci. 2007, 305, 366.

22. Tural B, Ozkan N and Volkan M J. Phys. Chem. Solids 2009, 70, 860. 


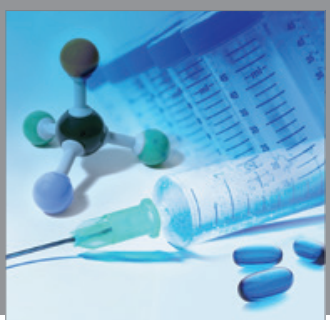

International Journal of

Medicinal Chemistry

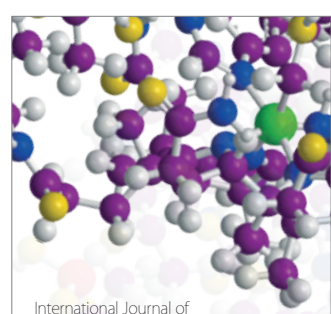

Carbohydrate Chemistry

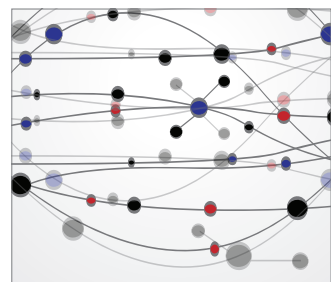

The Scientific World Journal
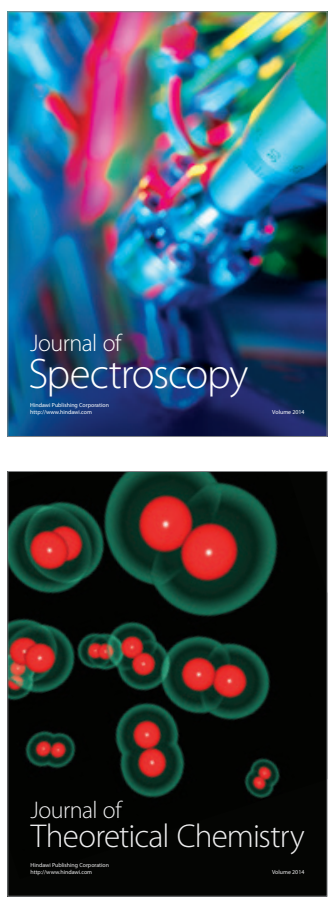
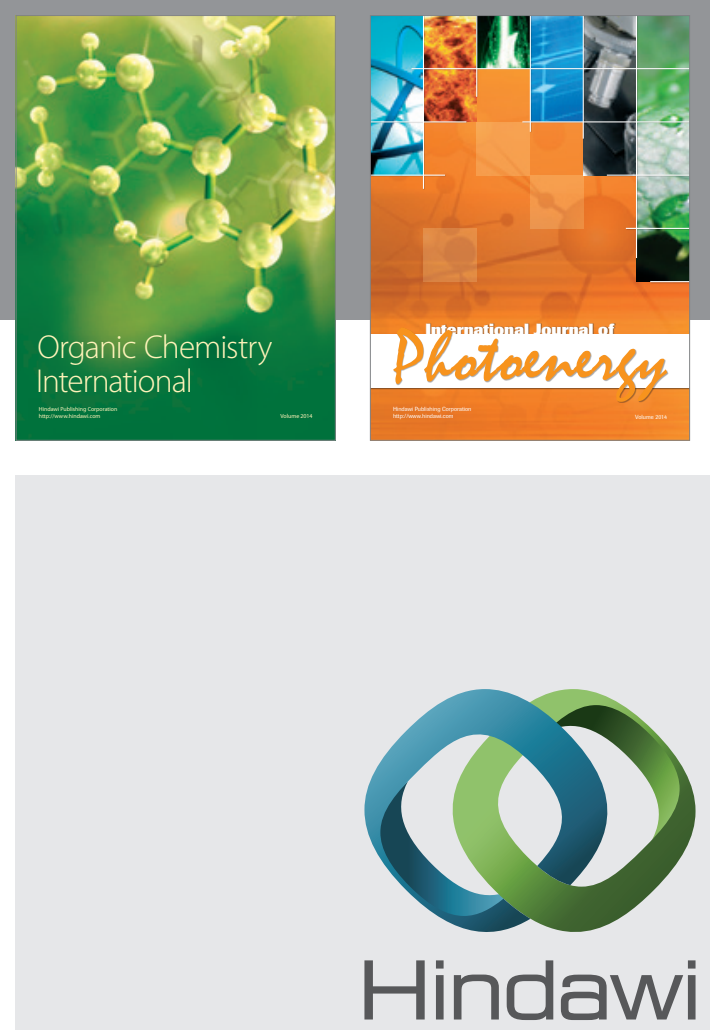

Submit your manuscripts at

http://www.hindawi.com
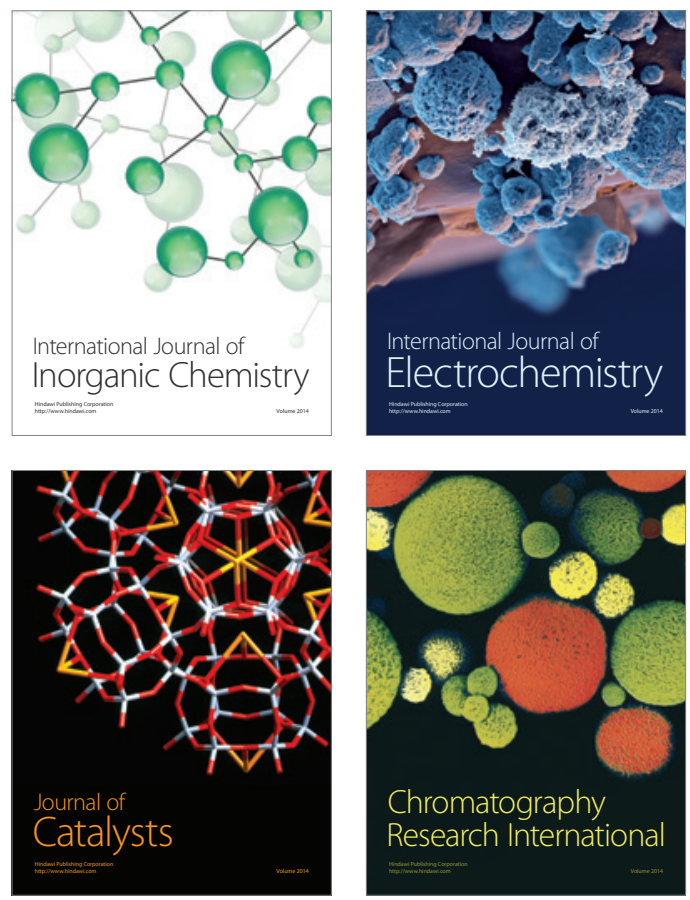
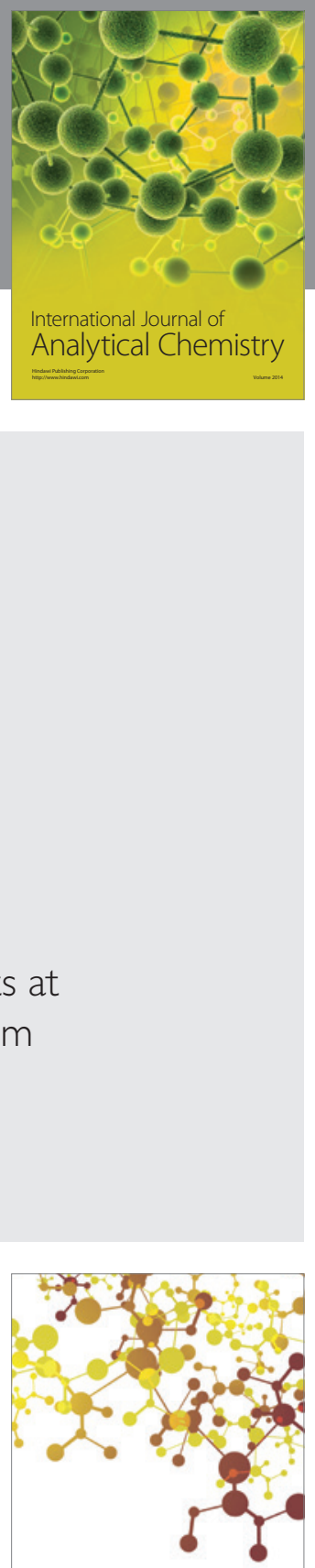

Journal of

Applied Chemistry
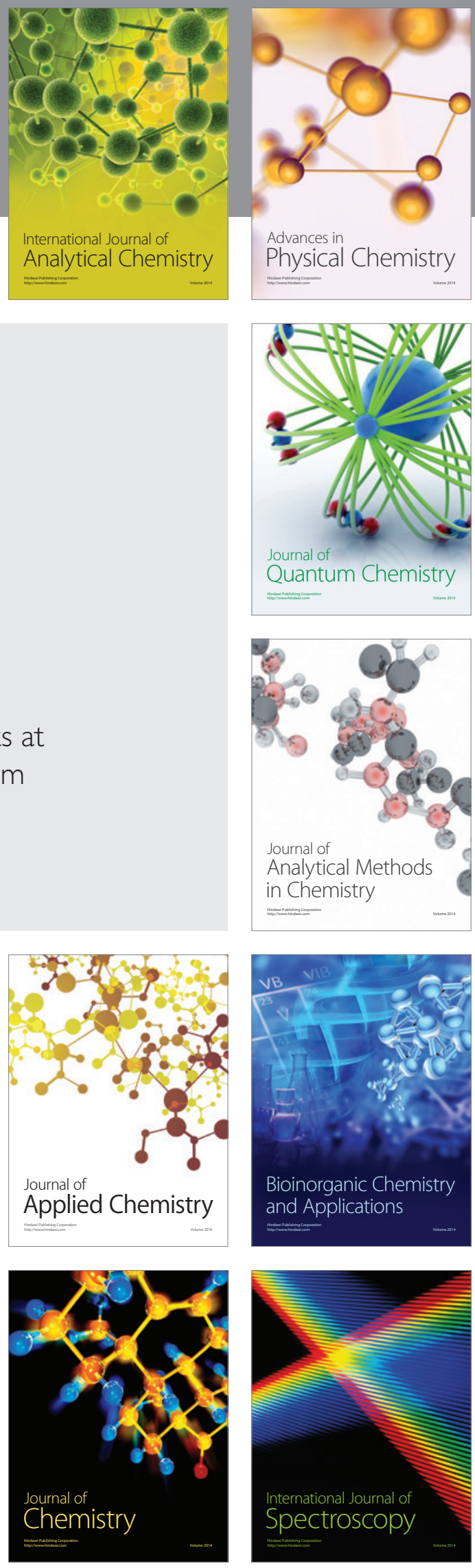